\title{
Zum 30-jährigen Klinikjubiläum von Dr. Norbert Frings
}

Im Oktober 2018 feiert Dr. med. Norbert Frings sein 30jähriges Dienstjubiläum als Chefarzt und Ärztlicher Direktor der Capio Mosel-Eifel-Klinik, Fachklinik für Venenerkrankungen und Rezidivoperationen, in Bad Bertrich. Mit seinem Namen eng verknüpft sind die Etablierung einer Schwerpunktversorgung von Venenpatienten als einer der Ersten in Deutschland, die Perfektionierung der Operationstechnik bei der Stammveneninsuffizienz sowie die wissenschaftliche Betrachtung des Phänomens der Neoangiogenese.

Norbert Frings wurde am 14. Januar 1949 in Essen geboren. Nach dem Medizinstudium in Köln und Düsseldorf begann er 1976 seine chirurgische Facharztweiterbildung, die ihn über Duisburg, Lüdenscheid und die Universitäten Marburg und Gießen führte. Es schlossen sich Tätigkeiten an als chirurgischer Oberarzt und im Rahmen der gefäßchirurgischen Weiterbildung in Essen und GelsenkirchenBuer.

Als frischgebackener Facharzt für Gefäßchirurgie trat Nobert Frings dann am 01.10.1988 die chefärztliche Leitung der Mosel-Eifel-Klinik in Bad Bertrich an. Zu dieser Zeit war die Mosel-Eifel-Klinik, idyllisch in einem Seitental der Mosel gelegen, in einem historischen Kurort unweit von Cochem entfernt, mehr oder weniger eine reine „Verödungsklinik“. Unter der Leitung von Norbert Frings erfuhr das Haus eine chirurgische Neuausrichtung, die es bis heute prägt und trägt, ohne dass man sich vor Innovationen - endovenöse Therapie, CHIVA, Schaumsklerosierung, extraluminale Valvuloplastie - verschlossen hätte. Mit einer jährlichen stationären Versorgung von bis zu 4000 Patienten zählt die Mosel-Eifel-Klinik bis heute zu den größten Venenfachkliniken Deutschlands. Unter seiner Leitung wurden mehr als fünfzig Phlebologen - zu denen sich auch der Autor zählen und glücklich schätzen darf auf einem sehr hohen Niveau und in der gesamten Bandbreite des Faches ausgebildet.
In den 30 Jahren unermüdlichen Schaffens war Norbert Frings vor allem von einer Idee, von einem Ideal, angetrieben, die Crossektomie zu perfektionieren. Die Operation der Stammvarikose galt zu Beginn seiner phlebologischen Tätigkeit als wenig effektives Therapieverfahren mit hohen Komplikations- und Rezidivraten. Nachdem er bereits zu Beginn der 1990erJahre die Tumeszenzlokalanästhesie als für die Patienten wenig eingreifendes Anästhesieverfahren in die Varizenoperation einführte und wissenschaftlich begleitete, galt sein besonderes Interesse in der Folgezeit u. a. der Aufarbeitung der Komplikationen nach Crossektomie und Stripping. Seine Analysen belegen eindrucksvoll, dass die offene Varizenoperation ein in hohem Maße sicheres Therapieverfahren ist. Etwa zeitgleich wuchs sein Interesse für die Rezidiv-Forschung, geprägt von dem nicht erschütterbaren Ziel, die Operationstechnik soweit zu verbessern, dass Crossenrezidive nicht oder nur noch in geringem Umfang auftreten. Durch seine Konzepte der Endothelinversionsnaht und der extensiven Crossektomie unter gleichzeitig schonender Präparationstechnik durch Anwendung der Tumeszenzlokalanästhesie ist die Varizenchirurgie diesem Ziel einen gewaltigen Schritt nähergekommen.

Mit mehr als 100 wissenschaftlichen Vorträgen auf nationalen und internationalen Kongressen und zahlreichen Publikationen in hochrangigen Journalen hat Norbert Frings in den vergangenen drei Dekaden vor allem die operative Phlebologie nach vorne gebracht, und das aus einer „nicht-universitären“ klinischen Tätigkeit heraus. Eine mehr als respektable Leistung. Seine Operationstechnik gilt heute vielen, nicht nur seinen zahlreichen Schülern, als Goldstandard.

Nach 30 Jahren Mosel-Eifel-Klinik nähert sich nun bald ein neuer Lebensabschnitt, den Norbert Frings mit seiner Ehefrau Dagmar, seinen Kindern und Enkelkindern, mit ebenso viel Sinn und Leben

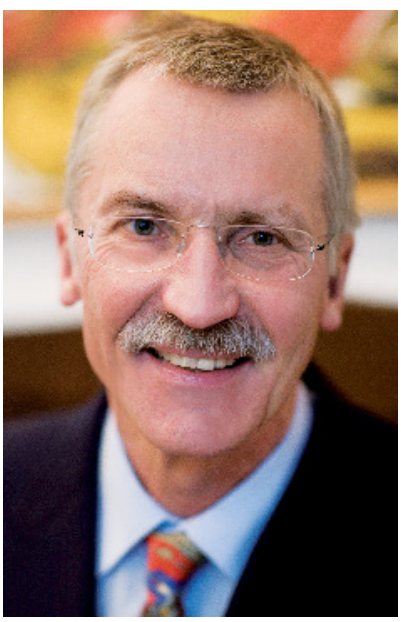

Dr. Norbert Frings füllen wird, wie seine berufliche und wissenschaftliche Karriere.

Zum 30-jährigen Klinikjubiläum gratuliere ich Ihm, auch im Namen der Deutschen Gesellschaft für Phlebologie, sehr herzlich und wünsche ihm ein erfülltes Verweilen mit stolzem Blick auf das Erreichte und für die Zukunft viel Freude und die beste Gesundheit!

\section{Knuth Rass, Simmerath}

